\title{
SCINTILLATION ARCS IN LOW-FREQUENCY OBSERVATIONS OF THE TIMING-ARRAY MILLISECOND PULSAR PSR J0437-4715
}

\author{
N. D. R. Bhat ${ }^{1,2}$, S. M. Ord ${ }^{1,2}$, S. E. Tremblay ${ }^{1,2}$, S. J. McSweeney ${ }^{1,2}$, and S. J. Tingay ${ }^{1,2}$ \\ ${ }^{1}$ International Centre for Radio Astronomy Research, Curtin University, Bentley, WA 6102, Australia \\ 2 ARC Centre of Excellence for All-sky Astrophysics (CAASTRO), Australia \\ Received 2015 October 1; accepted 2015 December 28; published 2016 February 9
}

\begin{abstract}
Low-frequency observations of pulsars provide a powerful means for probing the microstructure in the turbulent interstellar medium (ISM). Here we report on high-resolution dynamic spectral analysis of our observations of the timing-array millisecond pulsar PSR J0437-4715 with the Murchison Widefield Array (MWA), enabled by our recently commissioned tied-array beam processing pipeline for voltage data recorded from the high time resolution mode of the MWA. A secondary spectral analysis reveals faint parabolic arcs akin to those seen in high-frequency observations of pulsars with the Green Bank and Arecibo telescopes. Data from Parkes observations at a higher frequency of $732 \mathrm{MHz}$ reveal a similar parabolic feature with a curvature that scales approximately as the square of the observing wavelength $\left(\lambda^{2}\right)$ to the MWA's frequency of $192 \mathrm{MHz}$. Our analysis suggests that scattering toward PSR J0437-4715 predominantly arises from a compact region about 115 pc from the Earth, which matches well with the expected location of the edge of the Local Bubble that envelopes the local Solar neighborhood. As well as demonstrating new and improved pulsar science capabilities of the MWA, our analysis underscores the potential of low-frequency pulsar observations for gaining valuable insights into the local ISM and for characterizing the ISM toward timing-array pulsars.
\end{abstract}

Key words: instrumentation: interferometers - methods: observational - pulsars: general - pulsars: individual (PSR J0437-4715)

\section{INTRODUCTION}

Pulsar signals are subjected to a range of delays, distortions, and amplitude modulations from dispersive and scattering effects due to the ionized interstellar medium (ISM). These propagation effects scale steeply with the observing frequency, making low frequencies ( $\lesssim 400 \mathrm{MHz}$ ) less appealing for highprecision timing experiments such as pulsar timing arrays (PTAs; van Haasteren et al. 2011; Demorest et al. 2013; Manchester et al. 2013). However with PTA experiments approaching timing precisions of $\sim 0.1-0.8 \mu \mathrm{s}$, there is renewed interest in understanding the local ISM and its effects on highprecision timing, which may potentially limit achievable timing precision for most PTA pulsars (Arzoumanian et al. 2015; Lentati et al. 2015; Shannon et al. 2015). While the effects of temporal variations in dispersion measure (DM) have been investigated to a certain extent (You et al. 2007; Cordes \& Shannon 2010; Keith et al. 2013; Lee et al. 2014; Cordes et al. 2015; Lam et al. 2015), there exists only a limited understanding of the impact of scattering on timing precision.

PTA experiments currently rely on millisecond pulsars (MSPs) with low to moderate DMs $\left(\lesssim 50 \mathrm{pc} \mathrm{cm}^{-3}\right)$ to minimize ISM effects on timing precision. Recent work that used the Parkes Pulsar Timing Array (PPTA) data to place a limit on the strength of the stochastic gravitational wave background (Shannon et al. 2015) advocates shorter-wavelength $(\gtrsim 3 \mathrm{GHz}$ ) observations to alleviate ISM effects. However this is not currently feasible for the majority of PTA pulsars, for which $\sim 1-2 \mathrm{GHz}$ remains the most practically viable choice due to sensitivity limitations of existing telescopes and instrumentation. The ISM effects, including multipath scattering, may still be significant at those frequencies. Since scattering delays $\left(\tau_{\mathrm{d}}\right)$ scale steeply with the frequency $\left(\tau_{\mathrm{d}} \propto \nu^{-4}\right.$, where $\nu$ is the observing frequency; Bhat et al. 2004), they are more readily measurable in observations with new low-frequency arrays such as the Long Wavelength Array (LWA; Taylor et al. 2012), the Low Frequency Array (LOFAR; van Haarlem et al. 2013), and the Murchison Widefield Array (MWA; Tingay et al. 2013). Early pulsar observations with these instruments already demonstrate their potential in this direction (Dowell et al. 2013; Archibald et al. 2014; Bhat et al. 2014).

Observations of "scintillation arcs"-faint, parabolic arc-like features seen in secondary spectral analysis of pulsar observations-have provided new insights into both the microstructure of the ISM and the interstellar scattering phenomenon (Cordes et al. 2006; Rickett 2007; Stinebring 2007). First recognized by Stinebring et al. (2001) in Arecibo data, detailed studies of these arcs have revealed a variety and richness in their observational manifestations; e.g., forward and reverse arcs, and a chain of arclets (Hill et al. 2005; Putney \& Stinebring 2006; Brisken et al. 2010), which also stimulated a great deal of theoretical and modeling work (Walker et al. 2004; Cordes et al. 2006; Brisken et al. 2010). In the context of PTAs Hemberger \& Stinebring (2008) measured scattering delays from their observations of scintillation arcs in PSR B1737+13 $\left(\mathrm{DM}=48.9 \mathrm{pc} \mathrm{cm}^{-3}\right)$; the delays varied from $\sim 0.2$ to $2 \mu \mathrm{s}$ at a frequency of $1.3 \mathrm{GHz}$.

In this paper we present our observations of parabolic scintillation arcs in PSR J0437-4715, a high-priority target for PTAs. Details on processing and analysis are described in Sections 2 and 3 while in Sections 4 and 5 we describe the estimation of the arc curvature and the placement of the scattering screen. Our conclusions and future prospects are summarized in Section 6.

\section{OBSERVATIONAL DATA}

The MWA data used in this paper were recorded with the voltage capture system (VCS) developed for the MWA. The 

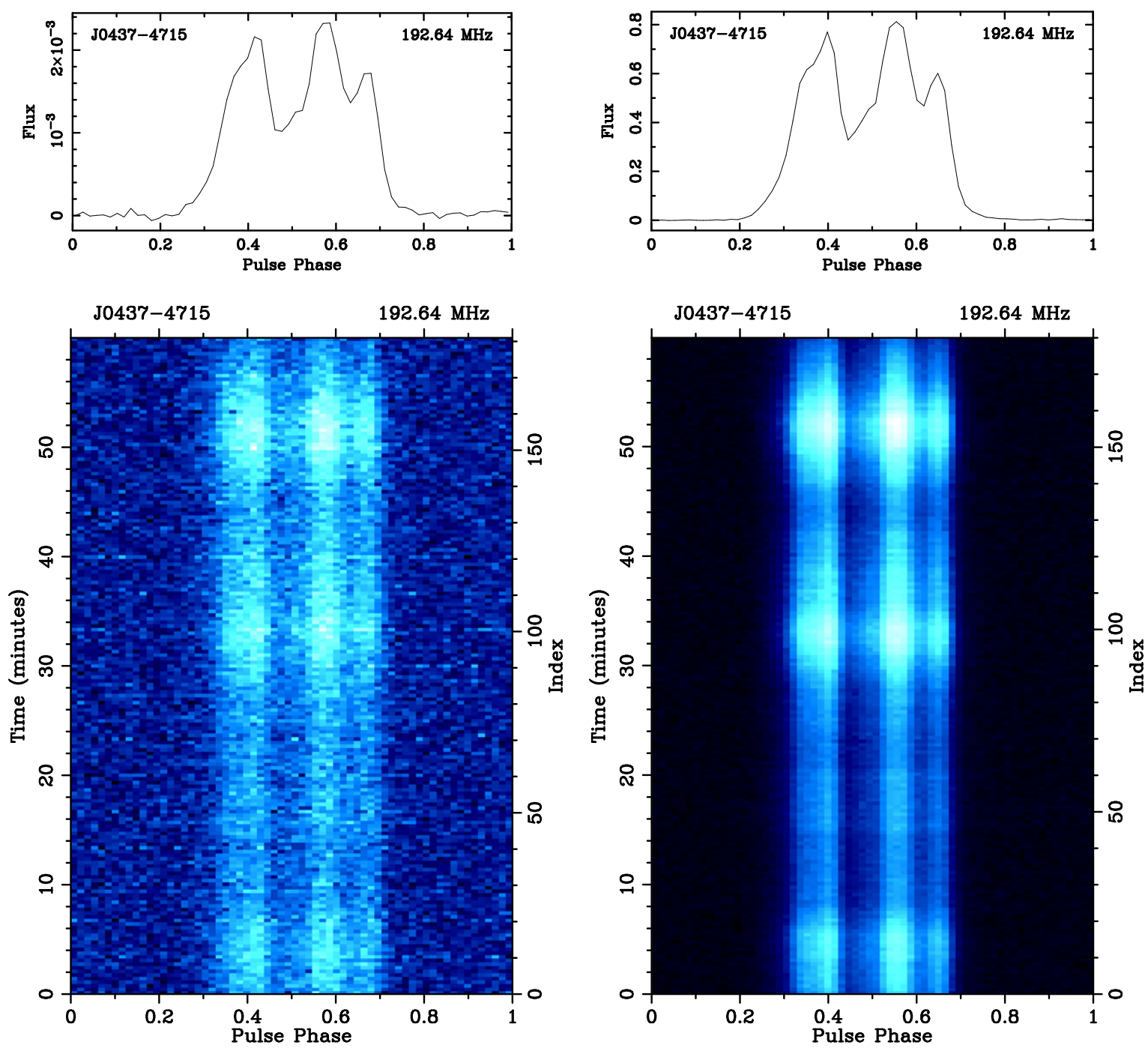

Figure 1. MWA observations of PSR J0437-4715 at a frequency of $192 \mathrm{MHz}$ over a time duration of $1 \mathrm{hr}$ and a bandwidth of $15.36 \mathrm{MHz}$. Left: incoherent addition of detected powers from all 128 tiles. Right: after reprocessing with the tied-array beamformer pipeline. Top: integrated pulse profiles. Bottom: pulse strength vs. time and pulse phase. The pulse phase resolution is approximately $90 \mu \mathrm{s}$. The improvement in $\mathrm{S} / \mathrm{N}$ is over an order of magnitude.

VCS functionality allows recording up to $24 \times 1.28 \mathrm{MHz}$ from all 128 tiles (both polarizations) at the native $100-\mu \mathrm{s}, 10-\mathrm{kHz}$ resolutions for up to $\sim 1.5 \mathrm{hr}$ (Tremblay et al. 2015), and is the primary observing mode for observations of pulsars and fast transients. Only half the recording capability $(12 \times 1.28 \mathrm{MHz})$ was available in the early days of VCS commissioning when our observations were performed $(\mathrm{MJD}=56559)$. Further details are described in Bhat et al. (2014). These data have now been reprocessed using our new beamformer pipeline that coherently combines voltage signals from all 128 tiles. The Parkes data used are from observations made at a frequency of $732 \mathrm{MHz}$ at an epoch two weeks later (MJD $=56573$ ) than our MWA observations.

\subsection{Tied-array Beam Processing of MWA Observations}

A tied-array beam is a coherent sum of voltage signals from individual tiles and is theoretically expected to yield a sensitivity improvement of $\sqrt{N_{\text {tile }}}$ over the incoherent addition of detected powers, where $N_{\text {tile }}$ is the number of tiles. For the MWA this means potentially an order of magnitude boost in sensitivity besides enabling high time resolution polarimetric studies. It involves incorporating delay models to account for the geometric and cable lengths as well as calibration for complex gains (amplitude and phase) of individual tiles and proper accounting for the beam models that incorporate polarimetric response of individual tiles. The calibration and beam information is provided by an offline version of the real-time calibration and imaging system, RTS (D. A. Mitchell et al. 2016, in preparation), which uses the visibilities generated from an offline version of the MWA correlator (Ord et al. 2015). Calibration was performed using Pictor A, a bright in-beam source at $\sim 10^{\circ}$ offset from PSR J0437-4715. The full processing pipeline runs on the Galaxy cluster of the Pawsey supercomputing facility ${ }^{3}$ that also hosts the archival VCS data after transport from the MRO. Further details on implementation of this processing pipeline are described in S. M. Ord et al. (2016, in preparation) where we also present the first pulsar polarimetric observations with the MWA.

Figure 1 shows the improvement in signal-to-noise from this tied-array beam processing for PSR J0437-4715 observations.

www.pawsey.org.au 


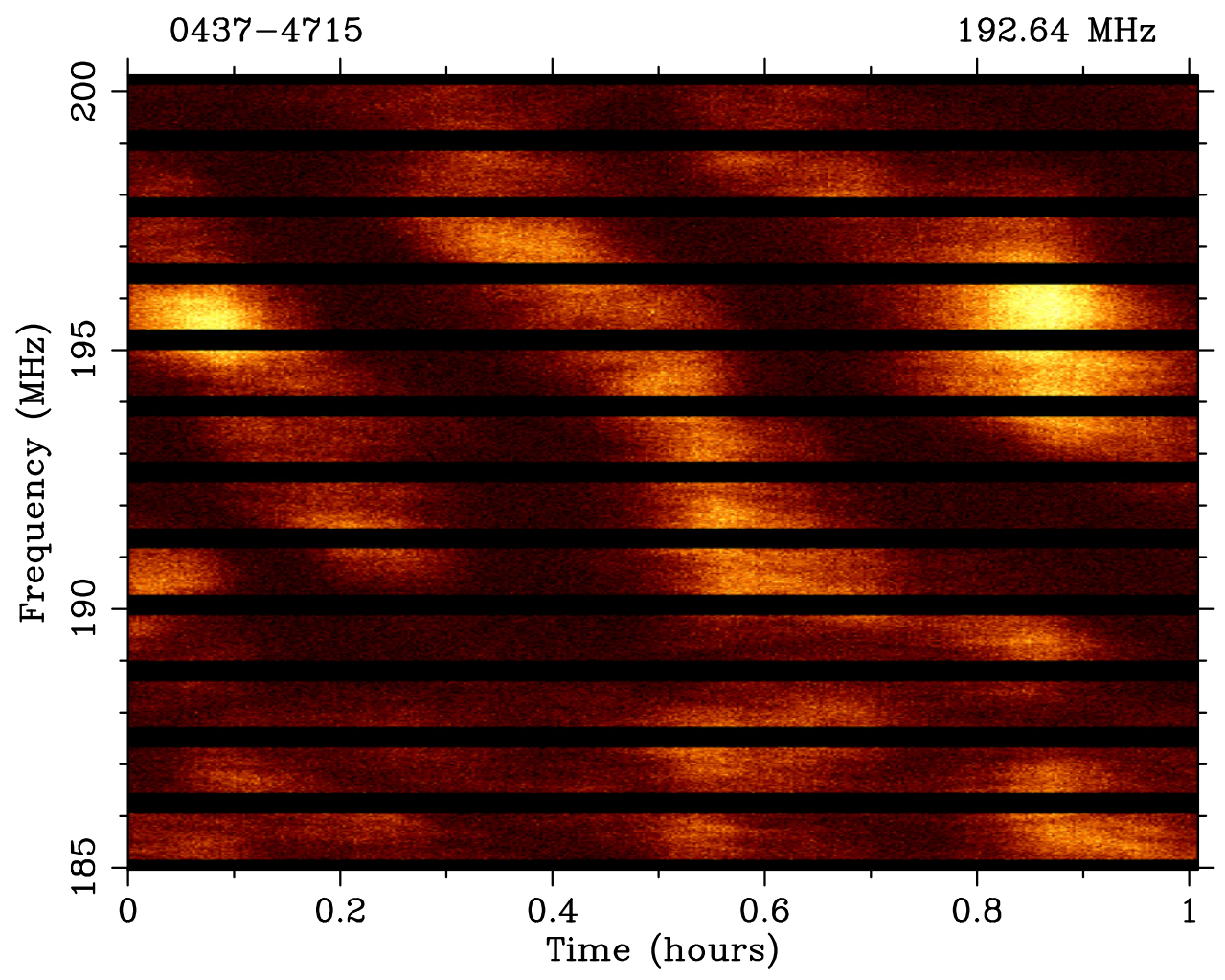

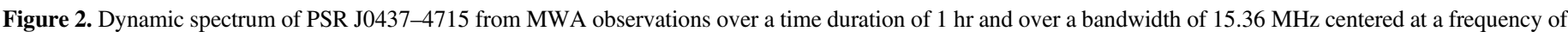

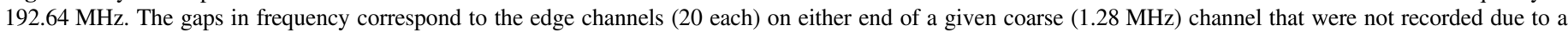
limitation in data recording in the early days of VCS commissioning. The data resolutions are $10 \mathrm{~s}$ in time and $10 \mathrm{kHz}$ in frequency.

The signal-to-noise ratio $(\mathrm{S} / \mathrm{N})$ of the integrated pulse profile has increased from $\sim 205$ (incoherent detection) to 2100 , i.e., an improvement of a factor of 10 , which is only $~ 10 \%$ less than the theoretical expectation for a coherent sum from 126 tiles. ${ }^{4}$ This translates to a mean $\mathrm{S} / \mathrm{N} \sim 3$ for individual pulses, however much larger values $(\mathrm{S} / \mathrm{N} \gtrsim 10)$ can be expected during the times of scintillation brightening.

\subsection{High-resolution Dynamic Spectra}

The beamformed data are processed using the DSPSR software package (van Straten \& Bailes 2011) to generate synchronously folded pulse profiles over 10-s sub-integrations. With the sensitivity improvement provided by the tied-array beam processing, we were able to generate a dynamic spectrum at the native frequency resolution of $10 \mathrm{kHz}$ for $\mathrm{VCS}$, a dramatic improvement over our earlier analysis that used a spectral resolution of $640 \mathrm{kHz}$ (Bhat et al. 2014). The resultant dynamic spectrum is shown in Figure 2. It is dominated by a small number of bright scintles whose intensity maxima drift in the time-frequency plane, a consequence of refraction through the ISM. The increased sensitivity and spectral resolution results in higher sensitivity to subtle features such as those caused by a combination of diffractive and refractive scattering effects.

Scintillation parameters including the characteristic scales in time and frequency (i.e., the scintillation bandwidth $\nu_{\mathrm{d}}$ and the diffractive time scale $\tau_{\text {diff }}$ ) can be obtained from a twodimensional auto-correlation function analysis of dynamic spectrum. The results from such an analysis along with a

\footnotetext{
4 Two tiles were excluded from tied-array beamforming owing to poor calibration solutions.
}

detailed comparison with the published measurements is presented in our earlier paper (Bhat et al. 2014). For the data in Figure 2 we obtain $\nu_{\mathrm{d}} \sim 1.7 \mathrm{MHz}, \tau_{\text {diff }} \sim 260 \mathrm{~s}$ and a drift rate (in the time-frequency plane) $d t / d \nu \sim 95 \mathrm{~s} \mathrm{MHz}^{-1}$ (with measurement uncertainties $\sim 25 \%$ ). Our measured $\nu_{\mathrm{d}}$ is discrepant with the majority of the published values, however it agrees with the larger scale of scintillation from Gwinn et al. (2006). Considering that all published measurements are from observations made at higher observing frequencies $(\sim 300-600 \mathrm{MHz})$, it is possible that many of them were underestimated, particularly when the observing bandwidth $(B)$ was not large enough to allow reliable measurements (e.g., $\nu_{\mathrm{d}} \gtrsim B$ ).

\section{SECONDARY SPECTRAL ANALYSIS}

The dynamic spectrum is a record of the pulse intensity as a function of time and frequency, $S_{1}(\nu, t)$, and is the primary observable for scintillation analysis. Its two-dimensional power spectrum is the secondary spectrum, $S_{2}\left(f_{\nu}, f_{\mathrm{t}}\right)=\left|S_{1}^{\dagger}(\nu, t)\right|^{2}$ (where $\dagger$ indicates two-dimensional Fourier transform). It is a powerful technique that captures interference patterns produced by different points in the image plane (e.g., Stinebring et al. 2001; Cordes et al. 2006). The scatter-broadened pulsar image is seen over a field of view $\sim D_{\mathrm{s}} \theta_{\text {diff }}$, where $D_{\mathrm{s}}$ is the effective distance to the screen and $\theta_{\text {diff }}$ is the half-width angular size of the broadened pulsar image. If $\boldsymbol{\theta}_{1}$ and $\boldsymbol{\theta}_{2}$ are two arbitrary points in the image plane the corresponding "fringe rates" in time and frequency, $f_{\mathrm{t}}$ and $f_{\nu}$, are given by

$$
f_{\mathrm{t}}=\frac{\left(\theta_{2}-\theta_{1}\right) \cdot \boldsymbol{V}_{\mathrm{eff}}}{s \lambda}, \quad f_{\nu}=\frac{D(1-s)\left(\theta_{2}^{2}-\theta_{1}^{2}\right)}{2 s c}
$$



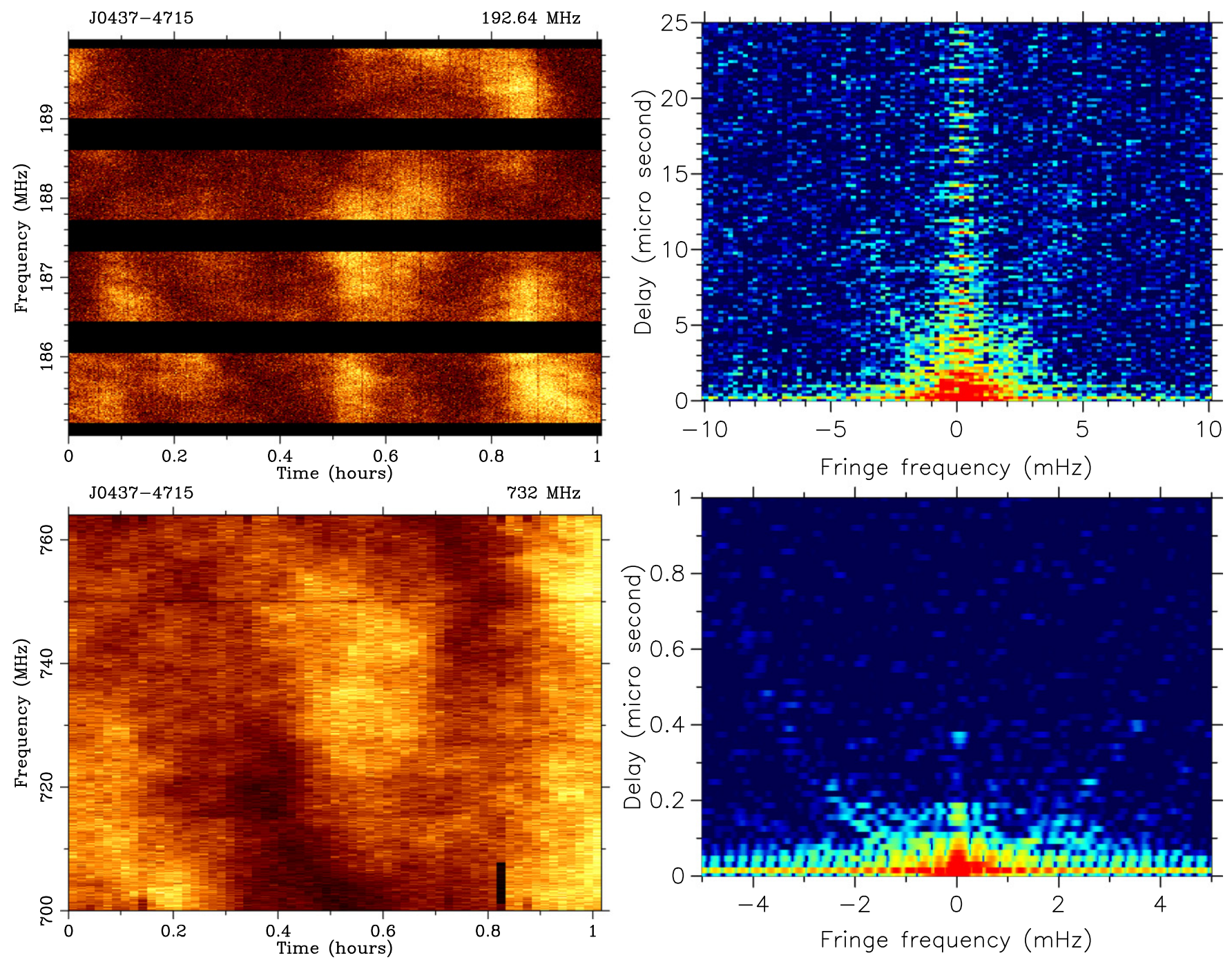

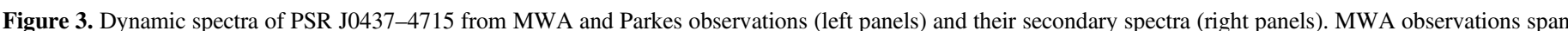

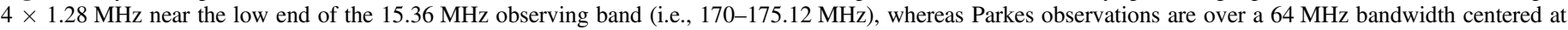

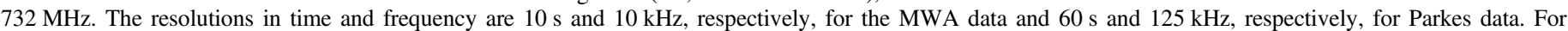

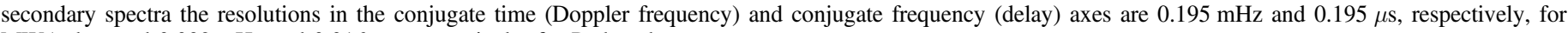
MWA data and $0.032 \mathrm{mHz}$ and $0.016 \mu \mathrm{s}$, respectively, for Parkes data.

where $c$ is the speed of light, $\lambda$ is the observing wavelength, and $s$ is the fractional distance of the screen from the source; $f_{\nu}$ is a measure of the differential time delay between pairs of rays and $f_{\mathrm{t}}$ is the temporal fringe frequency. Interference between the origin and pairs along an axis in the direction of net velocity vector $\boldsymbol{V}_{\text {eff }}$ produces parabolic scintillation arcs, represented by $f_{\nu}=\eta f_{\mathrm{t}}^{2}$. In essence, parabolic arcs can be described as a natural consequence of small-angle forward scattering.

\subsection{MWA Observations at $192 \mathrm{MHz}$}

Figure 3 shows the secondary spectrum from MWA observations at $192 \mathrm{MHz}$. There is a clear, albeit faint, arclike feature in the data, particularly on the left side of the zero Doppler frequency $\left(f_{\mathrm{t}}=0\right)$ axis. The feature is relatively more prominent in the lower one-third of the frequency band (170-175.12 MHz); however it is still visible-with somewhat reduced strength in data- over either a larger range or the fullfrequency range. Even though the full secondary spectrum spans fringe rates out to $50 \mathrm{mHz}$ and $50 \mu$ s for our dynamic spectral resolutions of $\Delta \nu=10 \mathrm{kHz}$ and $\Delta t=10 \mathrm{~s}$ in Figure 2, the arc feature visible is largely restricted to a small region $(\lesssim 5 \%)$ near the origin $\left(f_{\mathrm{t}} \lesssim 5 \mathrm{mHz} ; f_{\nu} \lesssim 15 \mu \mathrm{s}\right)$.

\subsection{Parkes Observations at $732 \mathrm{MHz}$}

To confirm the parabolic arc seen in MWA data we analyzed archival Parkes data from observations at $732 \mathrm{MHz}$, the only PPTA observing frequency that is below the expected transition frequency $(\sim 1 \mathrm{GHz})$ for this pulsar. Figure 3 shows the dynamic spectrum from observations made at an epoch two weeks later than MWA observations. The data were recorded with the ATNF Parkes Digital Filterbank (DFB4) and preprocessed over 1-minute sub-integrations and have a spectral resolution of $125 \mathrm{kHz}$. A parabolic arc feature is clearly visible in the secondary spectrum of these data (Figure 3). There are also hints of a "filled parabola," as seen in some of the published data at lower frequencies (e.g., Stinebring 2007). 

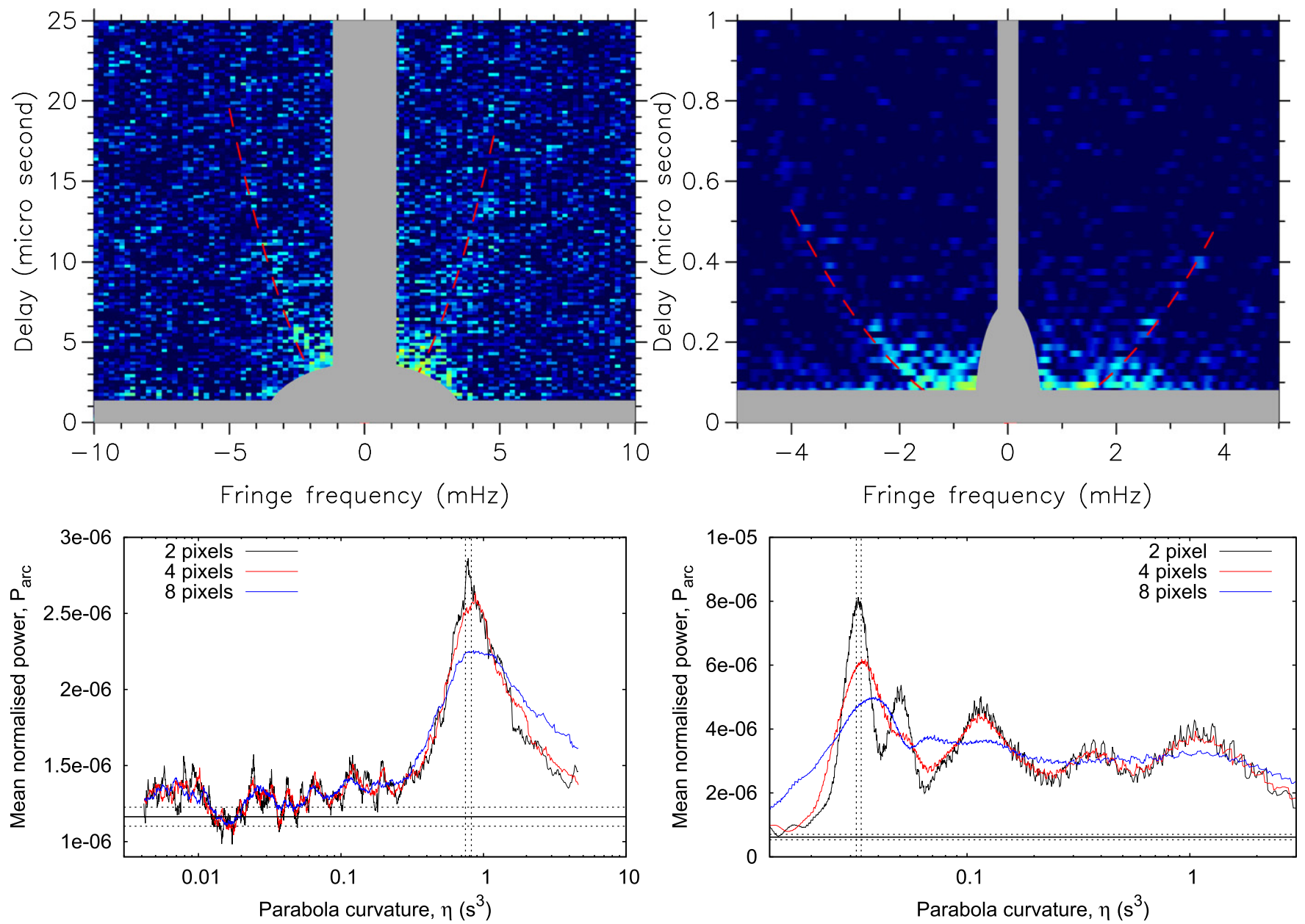

Figure 4. Top panels: secondary spectra of PSR J0437-4715 for MWA and Parkes data (Figure 3); the shaded areas (gray) were excluded from the analysis while estimating the arc curvature (dashed red curve). Bottom panels: mean arc strength $P_{\text {arc }}$ against the curvature parameter $\eta$ for MWA and Parkes observations (left and right panels, respectively) computed outside the excluded regions of low-frequency noise (gray in top panels). The 2, 4, and 8 pixels of the curves (in black, red, and blue, respectively) correspond to the size of the "smoothing window" (i.e., thickness of the parabola) used in the computation of $P_{\text {arc }}$. The solid and dotted horizontal lines correspond to the noise statistics estimated for a segment that is well outside the visible arc feature (see the text for details), whereas the dotted vertical lines correspond to the points where $P_{\text {arc }}$ is $1 \sigma$ below the peak (for the two-pixel curve).

\section{ARC CURVATURE AND THE PLACEMENT OF SCATTERING SCREEN}

Theoretical treatments on parabolic arcs and the scattering geometry are discussed by Stinebring et al. (2001) and Cordes et al. (2006; see also Brisken et al. 2010). The fringe frequency and delay parameters $f_{\nu}$ and $f_{\mathrm{t}}$ can be related to the curvature of the arc $(\eta)$, the pulsar distance $(D)$, proper motion $\left(V_{\mu}\right)$, and the placement of scattering screen $(s)$. As discussed by Cordes et al. (2006) this relation depends on the number of arcs seen in observations and the scattering geometry and in general, $s$ can be determined to within a pair of solutions that is symmetric about $s=1 / 2$. For a screen located at a fractional distance $s$ from the pulsar, the curvature parameter $\eta$ is given by

$$
\eta=\frac{D s(1-s) \lambda^{2}}{2 c V_{\mathrm{eff}}^{2} \cos ^{2} \alpha}
$$

where $D_{\mathrm{s}}=D s(1-s)$ is the effective distance to the screen and $\alpha$ is the angle between the net velocity vector $\boldsymbol{V}_{\text {eff }}$ and the orientation of the scattered image. The effective velocity $V_{\text {eff }}$ is the velocity of the point in the screen intersected by a straight line from the pulsar to the observer, which is the weighted sum of the pulsar's binary and proper motions, and the motion of the screen and the observer ( $V_{\text {scr }}$ and $V_{\text {Earth }}$ respectively). Its transverse component is given by

$$
\boldsymbol{V}_{\text {eff } \perp}=(1-s)\left(\boldsymbol{V}_{\mu \perp}+\boldsymbol{V}_{\text {bin } \perp}\right)+s \boldsymbol{V}_{\text {Earth } \perp}-\boldsymbol{V}_{\text {scr } \perp}
$$

where $V_{\mu \perp}$ is the transverse pulsar motion (i.e., proper motion) and $V_{\text {bin } \perp}$ is the pulsar's binary orbital motion (transverse component). Thus, the measurement of $\eta$ can be used to determine the location of the scatterer when all the contributing terms of Equation (3) (and hence the net $V_{\mathrm{eff} \perp}$ ) are precisely known.

The astrometric and binary orbital parameters are very welldetermined for PSR J0437-4715. Specifically, both the distance $(D)$ and the proper motion $\left(V_{\mu}\right)$ are known at very high precisions from timing and interferometric observations (Deller et al. 2008; Verbiest et al. 2008); a parallax measurement of $\pi=6.396 \pm 0.054$ yields $D=156.3 \pm 1.3$ pc, which, when combined with the proper motion measure-

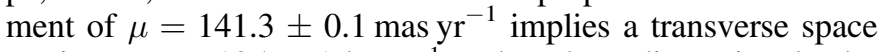
motion $V_{\mu}=105 \pm 1 \mathrm{~km} \mathrm{~s}^{-1}$. The three-dimensional sky geometry of the pulsar's binary orbit is also well-determined (van Straten et al. 2001; Verbiest et al. 2008), including the 
Table 1

Transverse Pulsar and Earth Motions ${ }^{\mathrm{a}}$ and the Estimated Screen Placements

\begin{tabular}{lccccr}
\hline \hline Observation & $\begin{array}{c}\text { Frequency } \\
(\mathrm{MHz})\end{array}$ & $\begin{array}{c}V_{\mu \perp} \\
\left(\mathrm{km} \mathrm{s}^{-1}\right)\end{array}$ & $\begin{array}{c}V_{\text {bin } \perp} \\
\left(\mathrm{km} \mathrm{s}^{-1}\right)\end{array}$ & $\begin{array}{c}V_{\text {Earth } \perp} \\
\left(\mathrm{km} \mathrm{s}^{-1}\right)\end{array}$ & $\begin{array}{c}\text { Arc Curvature, } \eta \\
\left(\mathrm{s}^{3}\right)\end{array}$ \\
\hline MWA & 192 & $(-55.2,-89.3)$ & $(18.9,1.1)$ & $(-21.97,18.19)$ & $0.78 \pm 0.02$ \\
Parkes & 732 & $(-55.2,-89.3)$ & $(-16.5,-6.8)$ & $(-26.18,12.81)$ & $0.033 \pm 0.001$ \\
\hline
\end{tabular}

Note.

${ }^{\mathrm{a}}$ The $x$ and $y$ components when projected onto the plane of sky with the $x$ axis along the line of nodes (see the text for details).

longitude of the ascending node $\Omega$. The screen velocity $\left(V_{\mathrm{scr}}\right)$ and the orientation angle $(\alpha)$ are generally unknown; ignoring these terms (i.e., assuming $V_{\text {scr }}$ is small compared to all other terms in Equation (3) and $\alpha=0$ ) leads to a simplified form for Equation (2) with the screen location $s$ being the sole unknown.

\subsection{Estimation of the Arc Curvature}

When observations are not limited by S/N (e.g., Arecibo data) and parabolic arcs are sharp and clearly visible, the curvature $\eta$ can be reliably estimated even as a best fit by eye (Stinebring et al. 2001; Hill et al. 2005). As the $\mathrm{S} / \mathrm{N}$ of our detections are comparatively lower, we adopt a new technique that is more systematic and robust. It employs feature extraction via the application of the (one-dimensional) generalized Hough transform. We essentially parameterize the parabolic feature by the curvature parameter $\eta$ and trial over a wide range of values within the constraints allowed by the data, each time summing the power along the parabolic segments outside the regions of low-frequency noise in order to minimize contamination from high spectral values near the origin and the zero axes. The mean power computed in this manner, $P_{\text {arc }}$ (henceforth referred to as "arc strength"), is given by

$$
P_{\operatorname{arc}}(\eta)=\frac{1}{N} \sum_{i=1}^{N} S_{2}\left(\eta f_{\mathrm{t}, \mathrm{i}}^{2}, f_{\mathrm{t}, \mathrm{i}}\right)
$$

where the summing procedure is performed along the points of arc outside the excluded low-frequency noise and out to delays beyond which little power is detectable (i.e., $i=1 \ldots N$, where $N$ corresponds to $f_{\nu}=20 \mu$ s for MWA data).

Figure 4 shows a plot of the arc strength $P_{\text {arc }}$ against $\eta$ from our analysis, where the point of maximum mean power corresponds to the best-fit curvature. ${ }^{5}$ We estimate $\eta=0.78 \pm 0.02 \mathrm{~s}^{3}$ for our MWA data (henceforth $\eta_{\text {mwa }}$ ) using this method. A similar analysis on Parkes data yields $\eta=0.033 \pm 0.001 \mathrm{~s}^{3}$ (henceforth $\eta_{\mathrm{pks}}$ ), where we also note multiple secondary peaks at larger values of $\eta$ (Figure 4), with a tendency for $P_{\text {arc }}$ to plateau $\sim 2 \mathrm{~dB}$ below the peak value. This is presumably arising from a sparse distribution of power inside the parabolic arc feature, suggesting scattered radiation arriving over a wider range of deflecting angles (and time delays). A closer examination of MWA data reveals hints of a similar case, albeit comparatively weaker, but seen as a slower tailingoff of $P_{\text {arc }}$ at larger values of $\eta$.

The measured values of $\eta_{\mathrm{mwa}}$ and $\eta_{\mathrm{pks}}$ do not scale as per the theoretically expected $\eta \propto \lambda^{2}$ relation. The implied scaling index of $\beta=-2.35 \pm 0.07$ (where $\eta \propto \lambda^{\beta}$ ) is larger than the values $(-1.8 \pm 0.1$ to $-2.1 \pm 0.1)$ that were determined by

\footnotetext{
5 The computation of $P_{\text {arc }}$ is restricted to $f_{\mathrm{t}}<0$ where the arc feature is more prominent.
}

Hill et al. (2003) from their observational data spanning a large frequency range $(0.4-2.2 \mathrm{GHz})$. As we explain below, this departure from the $\lambda^{2}$ scaling is due to the change in $V_{\text {eff } \perp}$ between the two observing epochs.

\subsection{Placement of the Scattering Screen}

To determine the location of the scatterer $(s)$ from the measurements of $\eta$, we need to compute the effective velocity $V_{\text {eff } \perp}$ at the two observing epochs (cf. Equations (2) and (3)). As the screen velocity $V_{\text {scr }}$ and the angle $\alpha$ are generally unknown, we assume $V_{\text {scr }} \sim 0$ and $\alpha \sim 0$ in our analysis. For the observing epochs of MWA and Parkes data $(\mathrm{MJD}=56559.878$ and $\mathrm{MJD}=56573.837$, respectively $)$, the corresponding true anomalies are $195^{\circ} .44$ and $350^{\circ} .76$, respectively, i.e., a difference in the orbital phase of 0.43 cycles. The transverse pulsar and binary motions, when projected onto the plane of the sky (with the $x$ axis defined along the line of nodes, positive toward the ascending node), are tabulated in Table 1 . There is a substantial change in $V_{\text {eff } \perp}$ between the two epochs due the pulsar's binary motion alone; accounting for just this term, i.e., $V_{\text {eff } \perp} \approx(1-s)$ $\left(\boldsymbol{V}_{\mu \perp}+\boldsymbol{V}_{\text {bin } \perp}\right)$, we obtain the fractional distance from the pulsar, $s=0.27 \pm 0.01$, from MWA measurements and $s=0.26 \pm 0.01$ from Parkes measurements.

The contribution from the Earth's orbital motion around the Sun $\left(V_{\text {Earth } \perp}\right)$ can also be significant depending on the pulsar's line of sight and the observing epoch although it will be weighted down for a screen that is located closer to the pulsar (Equation (3)). The $x$ and $y$ components of $V_{\text {Earth, } \perp}$ in the coordinate system that we have employed are tabulated in Table 1. The change in $V_{\text {Earth }}$ between the two observing epochs is relatively small $\left(\lesssim 5 \mathrm{~km} \mathrm{~s}^{-1}\right)$ in comparison to that from the pulsar's binary motion. Accounting also for this term involves solving for $s$ in Equation (2), which yields a pair of solutions, $s=0.26 \pm 0.01$ and $s=0.97 \pm 0.01$ from $\eta_{\text {mwa }}$, and $s=0.27 \pm 0.01$ and $s=0.98 \pm 0.01$ for $\eta_{\text {pks. }}$. However in light of our previous independent analysis based on scintillation measurements (Bhat et al. 2014), which yields a scintillation velocity $V_{\text {iss }}=325 \pm 90 \mathrm{~km} \mathrm{~s}^{-1} \sim 3 V_{\mu}$, i.e., $V_{\text {iss }}>V_{\mu}$ and therefore the solution suggesting a screen closer to the pulsar is favored. ${ }^{6}$ The implied screen placements are $115 \pm 2 \mathrm{pc}$ and $114 \pm 2 \mathrm{pc}$, respectively, from $\eta_{\mathrm{mwa}}$ and $\eta_{\mathrm{pks}}$ or effectively $115 \pm 3 \mathrm{pc}$ (from the Earth) if we combine the two estimates.

\section{DISCUSSION}

Our results in terms of the screen placements derived from MWA and Parkes observations are in very good agreement

\footnotetext{
$\overline{6 s=0.97 \text { would imply } V_{\text {iss }} \sim 0.03 V_{\mu}}$, which is not supported by observations.
} 
despite the fact that the observations were made at widely separated observing frequencies and not contemporaneous. The time separation of two weeks is significantly longer than the expected refractive time scale $\left(\tau_{\text {ref }}\right)$, i.e., the characteristic time in which a new volume of scattering material is expected to move across the pulsar's line of sight. It is given by $\tau_{\text {ref }} \sim\left(2 \nu_{\text {obs }} / \nu_{\mathrm{d}}\right) \tau_{\text {diff }}$, where $\nu_{\text {obs }}$ is the frequency of observation, and $\nu_{\mathrm{d}}$ and $\tau_{\text {diff }}$ are the scintillation bandwidth and diffractive time scale, respectively, both of which are measurable from dynamic spectra. For our MWA observations, $\nu_{\mathrm{d}} \sim 1.7 \mathrm{MHz}$ and $\tau_{\text {diff }} \sim 4.5$ minutes (Bhat et al. 2014), and hence $\tau_{\text {ref }} \sim 17 \mathrm{hr} \lesssim 1$ day. However, this estimate is not so reliable since it is based on single-epoch measurements, but even then it is unlikely $\tau_{\text {ref }}$ may be longer than $\sim$ a few days at the MWA's frequency. Our observational results may therefore suggest that the underlying scattering structure persists over multiple refractive cycles. The existence of such large scattering structures in the ISM was also suggested by past observations including those where the drift slopes and multiple imaging episodes were seen to persist over time scales of $\sim$ several months (e.g., Gupta et al. 1994; Rickett et al. 1997; Bhat et al. 1999).

Another subtlety pertains to the ISM volume sampled by multipath scattering which is a strong function of the observing frequency. As discussed in Section 3, the scatter-broadened pulsar image has a characteristic size $\sim D_{\mathrm{s}} \theta_{\text {diff }}$, and consequently the ISM sampled by MWA observations is more than two orders of magnitude larger than Parkes observations (since $\left.\theta_{\text {diff }} \propto \lambda^{2}\right)$. Although often ignored in observational interpretations, this can be a potentially important effect. Recent work of Cordes et al. (2015) explores this in great detail in the context of frequency-dependent (chromatic) DMs in timing-array observations. Nonetheless, it is not yet clear how this may influence scattering and scintillation observables and their scalings with the frequency. There is no compelling observational evidence in support of chromatic DMs, and a wealth of observational data on scintillation and scattering measurements are seen to follow the expected frequency scaling over a large range. In particular we note the work of Hill et al. (2003), who experimentally verified the scaling relation for scintillation arcs; their derived scaling indices range from $-1.8 \pm 0.1$ to $-2.1 \pm 0.1$ and are consistent with the $\lambda^{2}$ scaling despite observational data spanning a large frequency range (from 0.4 to $2.2 \mathrm{GHz}$ ). Contemporaneous observations at multiple different frequencies similar to those advocated by Lam et al. (2015) for improved DM corrections in PTA observations will be useful for gaining further insights into this aspect.

Aside from these subtleties our observations of scintillations arcs are clear indications of scattering toward PSR J0437-4715 arising from a localized region (thin screen). The implied screen location of $115 \pm 3 \mathrm{pc}$ is, incidentally, consistent with the expected location of $\sim 100-120 \mathrm{pc}$ to the edge of the Local Bubble (Snowden et al. 1990; Bhat et al. 1998; Cordes \& Lazio 2002; Spangler 2009). The possibility of the screen being closer to the pulsar was also hinted at by our earlier independent analysis in which our measured scintillation velocity $\left(V_{\text {iss }}=325 \pm 90 \mathrm{~km} \mathrm{~s}^{-1}\right.$ ) suggested a screen location of $\sim 80-120$ pc from the Earth (i.e., $s \sim 0.25-0.5$ ) based on $V_{\text {iss }} / V_{\mu} \sim 3$ (Bhat et al. 2014). Scattering toward PSR J0437-4715, therefore, most likely dominated by the material near the edge of the bubble.
Even as our observations of scintillation arcs suggest a small fraction of the scattered radiation arrive at large delays, its impact on timing precision may be negligibly small for this pulsar at its timing frequencies of $\sim 1-3 \mathrm{GHz}$. This is because PSR J0437-4715 is a weakly scattered pulsar with the second lowest value for the measured strength of scattering (the wavenumber spectral coefficient $\overline{C_{\mathrm{n}}^{2}} \sim 9 \times 10^{-5} \mathrm{~m}^{-20 / 3}$ from our measurement of scintillation bandwidth). Based on MWA observations, a transition to weak scintillation can be expected near $\sim 1 \mathrm{GHz}$ and consequently scattering effects are no longer relevant at frequencies $\gtrsim 1 \mathrm{GHz}$. However, this will not be the case for many other PTA pulsars. The DM range of PTA pulsars extends out to $\sim 300 \mathrm{pc} \mathrm{cm}^{-3}$ even though the majority of them are at DMs $\lesssim 50 \mathrm{pc} \mathrm{cm}^{-3}$. Since scattering delays $\left(\tau_{\mathrm{d}}\right)$ are expected to scale as $\mathrm{DM}^{2.2}$, timing perturbations $\sim 100 \mathrm{~ns}$ can be expected for PTA pulsars at the $\sim 1-2 \mathrm{GHz}$ timing frequencies. DM variations may still be the dominant source of ISM noise in PTA data; however, scattering effects may also be important, particularly if DM corrections are to rely on observations at frequencies $\lesssim 1 \mathrm{GHz}$. Observations at the low frequencies with MWA, LWA, LOFAR, and eventually SKALOW, can therefore prove to be very useful in assessing the importance of scattering delays and the nature of turbulent ISM toward PTA pulsars.

\section{CONCLUSIONS AND FUTURE WORK}

A new processing pipeline for MWA high time resolution data enables forming a coherent combination of tile powers from recorded voltages, bringing an order of magnitude improvement in the sensitivity for pulsar observations. We have demonstrated one of its applications through highresolution dynamic spectral studies of PSR J0437-4715 from MWA observations at $192 \mathrm{MHz}$. A secondary spectral analysis reveals parabolic scintillation arcs whose curvature scales as $\lambda^{2}$ to Parkes observations at $732 \mathrm{MHz}$ once accounted for the change in the net effective velocity due to the pulsar's binary orbital and the Earth's motions. Our analysis suggests that scattering toward PSR J0437-4715 predominantly arises from a compact region located about $\sim 115 \mathrm{pc}$ from the Earth, which is comparable to the distance to the edge of the Local Bubble $(\sim 100-120 \mathrm{pc})$ that encapsulates the local Solar neighborhood. Dedicated observational campaigns at the low frequencies of MWA and LOFAR, preferably contemporaneously with timing-array observations made at higher frequencies, can be potentially promising for a detailed characterization of the ISM along the lines of sight and for assessing the sources of ISM noise in timing-array data.

We thank an anonymous referee for several insightful comments that helped improve the content and presentation of this paper. We also thank J.-P. Macquart, R. M. Shannon, M. Bailes, and H. Knight for several useful discussions. This scientific work makes use of the Murchison Radio-astronomy Observatory operated by CSIRO. We acknowledge the Wajarri Yamatji people as the traditional owners of the Observatory site. NDRB is supported by a Curtin Research Fellowship. Support for the operation of the MWA is provided by the Australian Government Department of Industry and Science and Department of Education (National Collaborative Research Infrastructure Strategy: NCRIS) under a contract to Curtin University administered by Astronomy Australia Limited. We acknowledge the iVEC Petabyte Data Store and the Initiative in 
Innovative Computing and the CUDA Center for Excellence sponsored by NVIDIA at Harvard University and support from the Centre for All-sky Astrophysics (CAASTRO) funded by grant CE110001020.

\section{REFERENCES}

Archibald, A. M., Kondratiev, V. I., Hessels, J. W. T., \& Stinebring, D. R. 2014, ApJL, 790, L22

Arzoumanian, Z., Brazier, A., Burke-Spolaor, S., et al. 2015, arXiv: 1508.03024

Bhat, N. D. R., Cordes, J. M., Camilo, F., Nice, D. J., \& Lorimer, D. R. 2004, ApJ, 605, 759

Bhat, N. D. R., Gupta, Y., \& Rao, A. P. 1998, ApJ, 500, 262

Bhat, N. D. R., Ord, S. M., Tremblay, S. E., et al. 2014, ApJL, 791, L32

Bhat, N. D. R., Rao, A. P., \& Gupta, Y. 1999, ApJS, 121, 483

Brisken, W. F., Macquart, J.-P., Gao, J. J., et al. 2010, ApJ, 708, 232

Cordes, J. M., \& Lazio, T. J. W. 2002, arXiv:astro-ph/0207156

Cordes, J. M., Rickett, B. J., Stinebring, D. R., \& Coles, W. A. 2006, ApJ, 637, 346

Cordes, J. M., \& Shannon, R. M. 2010, arXiv:1010.3785

Cordes, J. M., Shannon, R. M., \& Stinebring, D. R. 2015, arXiv:1503.08491

Deller, A. T., Verbiest, J. P. W., Tingay, S. J., \& Bailes, M. 2008, ApJL, 685, L67

Demorest, P. B., Ferdman, R. D., Gonzalez, M. E., et al. 2013, ApJ, 762, 94

Dowell, J., Ray, P. S., Taylor, G. B., et al. 2013, ApJL, 775, L28

Gupta, Y., Rickett, B. J., \& Lyne, A. G. 1994, MNRAS, 269, 1035

Gwinn, C. R., Hirano, C., \& Boldyrev, S. 2006, A\&A, 453, 595

Hemberger, D. A., \& Stinebring, D. R. 2008, ApJL, 674, L37

Hill, A. S., Stinebring, D. R., Asplund, C. T., et al. 2005, ApJL, 619, L171

Hill, A. S., Stinebring, D. R., Barnor, H. A., Berwick, D. E., \& Webber, A. B. 2003, ApJ, 599, 457
Keith, M. J., Coles, W., Shannon, R. M., et al. 2013, MNRAS, 429, 2161

Lam, M. T., Cordes, J. M., Chatterjee, S., \& Dolch, T. 2015, ApJ, 801, 130

Lee, K. J., Bassa, C. G., Janssen, G. H., et al. 2014, MNRAS, 441, 2831

Lentati, L., Taylor, S. R., Mingarelli, C. M. F., et al. 2015, MNRAS, 453, 2576

Manchester, R. N., Hobbs, G., Bailes, M., et al. 2013, PASA, 30, 17

Ord, S. M., Crosse, B., Emrich, D., et al. 2015, PASA, 32, e006

Putney, M. L., \& Stinebring, D. R. 2006, ChJAS, 6, 233

Rickett, B. J. 2007, in ASP Conf. Ser. 365, SINS-Small Ionized and Neutral Structures in the Diffuse Interstellar Medium, ed. M. Haverkorn \& W. M. Goss (San Francisco, CA: ASP), 207

Rickett, B. J., Lyne, A. G., \& Gupta, Y. 1997, MNRAS, 287, 739

Shannon, R. M., Ravi, V., Lentati, L. T., et al. 2015, Sci, 349, 1522

Snowden, S. L., Cox, D. P., McCammon, D., \& Sanders, W. T. 1990, ApJ, 354,211

Spangler, S. R. 2009, SSRv, 143, 277

Stinebring, D. 2007, in ASP Conf. Ser. 365, SINS-Small Ionized and Neutral Structures in the Diffuse Interstellar Medium ed. M. Haverkorn \& W. M. Goss (San Francisco, CA: ASP), 254

Stinebring, D. R., McLaughlin, M. A., Cordes, J. M., et al. 2001, ApJL, 549, L97

Taylor, G. B., Ellingson, S. W., Kassim, N. E., et al. 2012, JAI, 1, 1250004

Tingay, S. J., Goeke, R., Bowman, J. D., et al. 2013, PASA, 30, 7

Tremblay, S. E., Ord, S. M., Bhat, N. D. R., et al. 2015, PASA, 32, e005

van Haarlem, M. P., Wise, M. W., Gunst, A. W., et al. 2013, A\&A, 556, A2

van Haasteren, R., Levin, Y., Janssen, G. H., et al. 2011, MNRAS, 414, 3117 van Straten, W., \& Bailes, M. 2011, PASA, 28, 1

van Straten, W., Bailes, M., Britton, M., et al. 2001, Natur, 412, 158

Verbiest, J. P. W., Bailes, M., van Straten, W., et al. 2008, ApJ, 679, 675

Walker, M. A., Melrose, D. B., Stinebring, D. R., \& Zhang, C. M. 2004, MNRAS, 354, 43

You, X. P., Hobbs, G., Coles, W. A., et al. 2007, MNRAS, 378, 493 